\title{
ORGANIC FUNCTIONAL GROUP ANALYSIS AND DRUG STABILITY
}

\author{
F. Pellerin, D. Baylocq and N. Chanon
}

Centre d'Etudes Pharmaceutiques de l'Universite de Paris Sud. Laboratoire de Chimie Analytique, Rue J.B. Clement, 92296 CHATENAY-MALABRY, France

\section{CONTENTS}

INTRODUCTION 171

1. GENERAL IDEAS 172

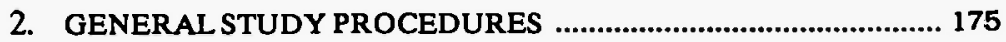

2.1. Separation of isomers 176

2.2. The role of radiation 177

2.3. Reactivity in solution 178

3. REACTIVITY IN COMPLEX MLXTURES 185 REFERENCES 193

\section{INTRODUCTION}

The study of the stability of drugs has many aspects and involves the participation of numerous specialists at all stages of the development of the drug, starting with its synthesis and development right up to its administration and elimination. From the regulatory standpoint, the objective of the stability study is to prove the maintenance of activity and to determine the conditions and duration of the shelf life of the drug. The pharmacologist and the toxicologist are concerned with verifying the consistency of the drug's activity and the absence of reactions or toxic phenomena. Specialists in its formulation are concerned with the maintenance of the technological and pharmacokinetic properties. The biologist needs to know the 
duration of the drug's activity after administration, its transformations and its metabolism. The analytical chemist intervenes in all these fields and at all stages, and must provide the means best adapted to the goal being pursued, from the identification of the active component and its concentration, to the determination of the normal and abnormal breakdown products formed during storage and which are inherent in the reactions due to external conditions, to the interaction of components of the pharmaceutical form, or to the biological medium.

Finding a common denominator for the analytical chemist for all the problems that are posed by stability studies, and attempting to infer some general ideas for the conduct of the research, is a particularly difficult task.

In fact, from whatever standpoint one views the stability of a drug, the purpose of this paper is to show that this study flows from the prior study of the reactivity; that is, the physical and chemical transformations that the drug undergoes under the action of various agents, chemicals and compounds. It comes prior ic fixing standards and specifications, the lifetime, and the study of biological availability and metabolism. To foresee the transformations and prevent the interactions is, therefore, a problem facing the analytical chemist, which he must try to solve using his experience and his intervention at all stages in the development of the drug.

After a general review, the reactivity of functional groups under the influence of external agents, various kinds of reagents or components of pharmaceutical formulations will be described with the help of examples taken from personal work.

\section{GENERAL IDEAS}

1.1 Reactivity seen from the standpoint of the physical, physicochemical properties of a part of the molecule, of a group of atoms or an atom, includes all the properties of the functional groups, and, through them, of the molecule itself. It also comprises the methods of identification and of measuring the concentration in 
medical formulations, i.e. methods that are based on the choice of a specific reaction or, at least, a selective reaction of the component being studies $/ 1,2 /$.

The reactivity is also, and especially, the study of the properties of functional groups, of the influence of other functional groups and of the size of the molecule. The results of this study are of great importance. In effect, it is evident that a functional group of low reactivity in the middle of the molecule will be little affected by its environment: external agents, excipients or solvents. Conversely, knowledge of the reactivity specific to each group, or imputable to their interactions, facilitates knowledge of the molecule's weak points. The analyst will carry out these reactions under various conditions and then try them on mixtures and on the drug itself. The stability of the drug is thus determined by the reactivity of the molecule and the identification of the breakdown products.

It should also be noted that the study of the reactivity of the molecule sometimes allows the metabolism of the drug to be predicted. The successive transformations that an organic molecule undergoes in vitro under the influence of various reagents or in conditions close to those in living tissues, allow a working hypothesis to be established for the prediction of the breakdown of the drug in the organism.

1.2 The stability is established on the basis of a set of data. The study of the behaviour of the raw material and of the drug itself in different environments (temperature, light, humidity) generally provides a basis for stability studies. Nevertheless, the objectives of this preliminary study and the interpretation of the results should not be misjudged. One of the objectives lies in the determination of "alterability". Some simple methods, such as the thin-layer chromatography recommended by Pesez $\beta$ / in a W.H.O. publication allow detection of alterations at the place of use, notably in a tropical environment, and thus to decide whether to use the drug or not. 
Moreover, these tests reveal the relative importance of these three factors in a chemical reaction: air, light and temperature. Light appears to be the most important factor, an increase in temperature only accelerates a process that has already been induced. The test of the effects of temperature increase should not be interpreted as a test of accelerated ageing or a means of fixing the shelf life, but as a test of the behaviour of the molecule. It indicates the possible breakdown products of the molecule. Some examples are given below.

1.3 Kinetics of chemical reactions. The study of chemical kinetics has an important place in the study of stability, as in biopharmacological or drug-metabolism studies. The role of chemical kinetics will not be developed here. The accelerated study of the stability of drugs and pharmaceutical preparations is based on the application of fundamental principles and theories of physical chemistry and chemical kinetics. It is based on Arrhenius' equation $\mathrm{K}=\mathrm{Ae}^{-\delta \mathrm{H} / \mathrm{RT}}$ where $\mathrm{A}$ is a constant related to the entropy of the reaction and $\delta \mathrm{H}_{a}$ is the heat of activation. whenever the relation at high temperature $(T)$ is linear, it is feasible to study the prediction of stability storage temperatures. The application of the laws of physical chemistry and kinetics, the subject of Garret's $/ 4 /$ work in this field, is now well known in the pharmaceutical industry. The interpretation of the stability of a chemical drug and the forecasting of its shelf life generally rest on a study of the active constituent in aqueous solution at high temperature; this is followed by the variation of the physicochemical properties such as the development of the infra-red absorption spectrum, and, frequently in the ultra-violet spectrum, that of an electrochemical property measured by the development of an intensity/potential curve or by the appearance of a breakdown product detected by chromatography.

It is not always sufficient to measure a property and its variations; the difficulty lies in knowing the development of the reaction and the nature of the products formed. The concentration 
of the active component measured by a spectral method does not always yield information on the stability, since the transformation may affect only a part of the molecule and the product formed may have spectral characteristics little different from those of the original component. The stability is determined by the concentration of breakdown products, assuming that the component does not itself undergo another reaction. Whenever several linear or convergent reactions develop, the relation between the kinetics at various temperatures and the shelf life becomes illusory and, consequently, the result of a physical measurement such as absorbance is uninterpretable because it represents, globally, the results of reactions for which the kinetics are different. Here again, knowledge of the reactivity and the transformations should precede kinetic studies.

Finally, studies under various conditions and the application of kinetic methods lead to a considerable number of data that are often hard to interpret. The reactivity of the functional groups will often allow a simplification and especially an explanation of the stability of the drug.

\section{GENERAL STUDY PROCEDURES}

A knowledge of the stability can be approached through a study of reactions conducted under various conditions, dry or in solution: the effect of temperature $\left(37^{\circ}-45^{\circ} \mathrm{C}\right)$ or of light in acid or alkaline media, or of oxidizing or reducing agents. The experimental transformation reveals the weak points of the molecule and facilitates the identification and determination of the structure of the components so formed. The results obtained by relatively aggressive means, direct the search for these same products after conservation of the finished product under conditions of use. They also allow chemically tolerable limits to the breakdown products to be fixed, before the toxicological problem is addressed. At the same time, the experimental transformation justifies the methodology of the study. 
2.1 Separation of the $E$ and $Z$ isomers. (Fig. 1). The synthesis of Diclofurime (I) leads to the formation of two isomers, $E$ (II) and $Z(I I I)$; the predominant isomer, $E(I)$ is the Diclofurime, which is separated by recrystallization, and its structure has been determined by NMR $/ 5 /$ and chemically $/ 6,2 /$. The reactivity of this component under various conditions is revealed by the

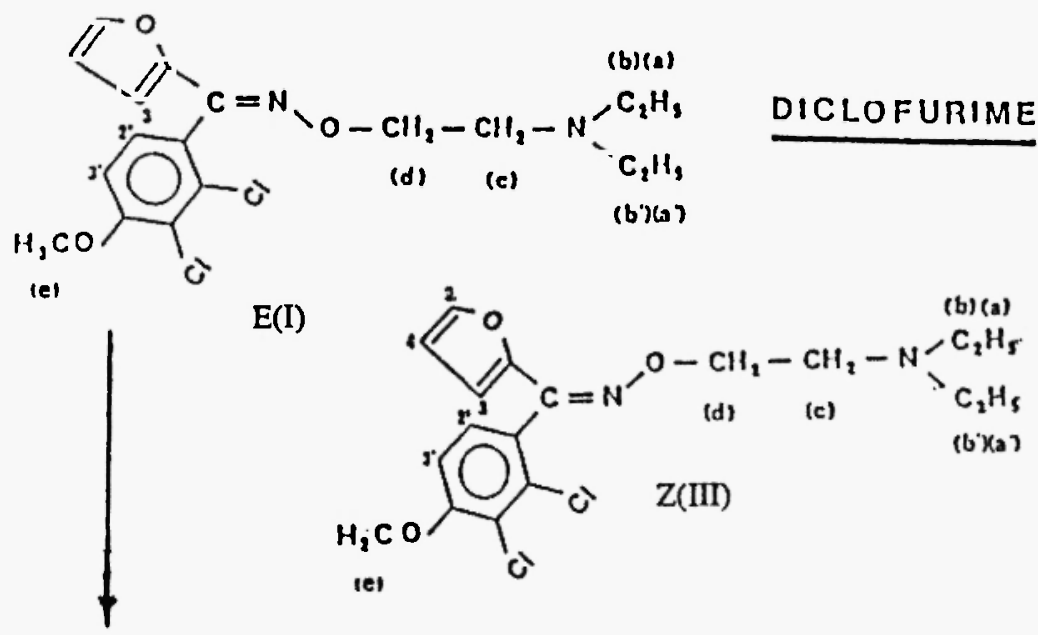<smiles>CCNCNC(C)(C)CON=C(c1ccco1)c1cc(OC)c(Cl)c(Cl)c1Cl</smiles><smiles>COc1ccc(C(=O)O)c(Cl)c1Cl</smiles> 
formation of the following products, which have been identified and the structure of which has been determined:

- loss of the ethyl group (dealkylation) (IV)

- rupture of the $\mathrm{N}$-oxime group with the formation of nitrile (V)

- usual hydrolysis of the nitrile group with the production of amide (VI) and acid (VII).

2.2 The role of radiation. The primordial roie of light radiation merits attention, since it results in very diverse reactions. the photo-transformation of phenyl-3 butanone-2 oxime goes as follows, with the formation of nitrile $\Pi /$ :

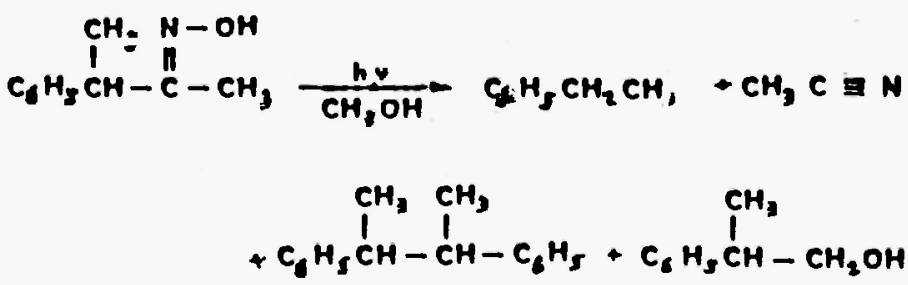

The photolysis of Pentobarbital causes the rupture of the pyrimidine ring, according to Barton /8/<smiles>CCCC1(CC)C(=O)NC(=O)NC1=O</smiles>

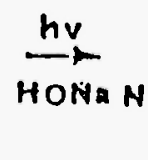<smiles>CCC(C)C(=O)NC(N)=O</smiles><smiles>CCC(CC(CC)C(=O)NCCCCCCC(=O)O)C(=O)O</smiles> 
The photo-oxidation of phenothiazine, with the formation of $\mathbf{N}$ and S-oxides, is well known and has been particularly studied by Heyes and Salmon in the case of fluphenazine and its esters $\mathbf{~} \%$. Sterilization by gamma rays is sometimes the cause of breakdown in packaging of medical/surgical equipment. It causes the modification of the mechanical and technological properties of the materials (solidity, colour, elasticity, etc.) and the products formed can be the cause of migration. Such is the breakdown of PVC followed by DTA. Sterilization by radiation can also affect additives in materials such as antioxidants. With $C$. Majcherczyck $/ 10,11 /$ we have shown, for example, the GOODRITE 3114 is broken down by Gamma rays; at first, hydroxyl groups are oxidized to form quinones (I); then the molecule is split to yield an isocyanurate II and finally an aldehyde (III), the nature and structure of which have been determined by mass spectrometry.

The importance of photochemical reactions implies the necessity of determining the stability, under the influence of light, of all organic drugs, by determining the reaction mechanisms and the nature of the products formed. Another consequence of the photosensitivity of drug molecules is research on protective materials. the choice of containers and packaging is particularly important especially in the oase of plastics which are themselves sensitive to light radiation or sterilizing radiations. this study is essential throughout the development phase of the drug.

\subsection{Reactivity in solution.}

2.3.1 The role of the solvent. The study of the reactivity should include tests in solution. The importance and the role of the solvent are well known to the analyst who exploits its properties for analytical purposes. The solvent intervenes through its power of dissolution and its involvement in chemical proton-exchange or redox reactions. the use of protonometry or redoximetry made by the analyst is well known in the study of different solvents. These reactions in solution are also applicable to the study of the stability of the molecule, and it 


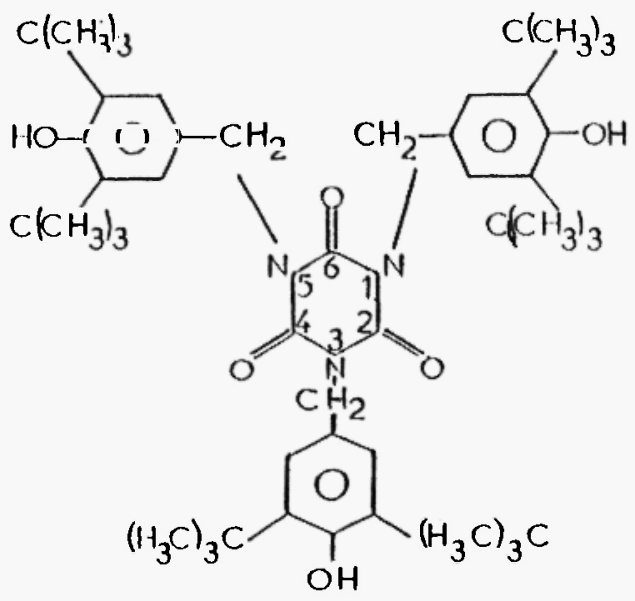

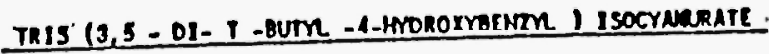
(GoOdRITE 3114)

(I) QUINONIC DERIVATE -HC=? $\begin{aligned} & \mathrm{C}\left(\mathrm{CH}_{3}\right)_{3} \\ & =0 \\ & \mathrm{C}\left(\mathrm{CH}_{3}\right)_{3}\end{aligned}$

(II) (z)ISUBITTUTED) I SOCYANIMRATE<smiles>Cc1cc(C[18F])cc(C)c1O</smiles>

(III) MLEHTOE

( 3-5-OI-TERT-BUTM-4-HOROXY-BERZNDEHOE)

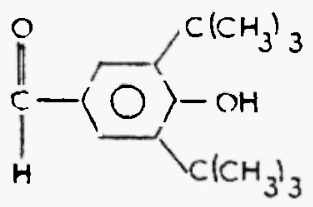


should not be forgotten that numerous liquid or semi-liquid pharmaceutical formulations enclose organic solvents that are far from being inert; polyethylene glycol, glycols, polysorbates, for example, change the $\mathrm{pH}$ of the solutions and have an effect on the reactions of the molecules. In suspensions or emulsions, the partition of the chemical component between these two phases affects the reactivity; the partition coefficient is, therefore, one of the measures of stability.

2.3.2 Cyclic reactions are common; glutamic acid can be cycled by heating in aqueous solution and pyrrolidoxycarboxylic acid. The breakdown of aspartam is also an example of a cyclic reaction that is well known in food chemistry; a derivative known as diketopipetazine is formed. The stability, like the purity, of this sweetener is not only linked to its content of cyclic derivatives; the breakdown ends, in effect, with the hydrolysis of the ester and amide groups, leading to phenylalanine and aspartic acid as shown in Fig. 2.

2.3.3 Equilibrium reactions between dissolved molecules and their breakdown products constitute essentially a factor of stability.

In aqueous solution, an equilibrium reaction is established between sodium noramidopyrine methane sulphonate (NAMS Na) and methylaminoantipyrine (MAA). Solutions kept away from light and air remain stable.

The extraction of MAA by chloroform to determine its concentration $/ 12,13 /$ immediately displaces the equilibrium. Also, the breakdown of NAMS $\mathrm{Na}$ follows two different routes, as shown in Fig. 3 : hydrolytic (a), according to $/ 14 /$, and oxidative (b), according to $/ 15 \%$. In any case, the breakdown leads to the formation of methylaminoantipyrine (MAA) with or without yellowing due to methylrubazonic acid. Gas-liquid chromatography /12/ or thin-layer chromatography or liquid-phase chromatography $/ 12 /$ allow the formation of these products to be followed and the stability to be monitored. The presence of MAA is pharmaceutical 

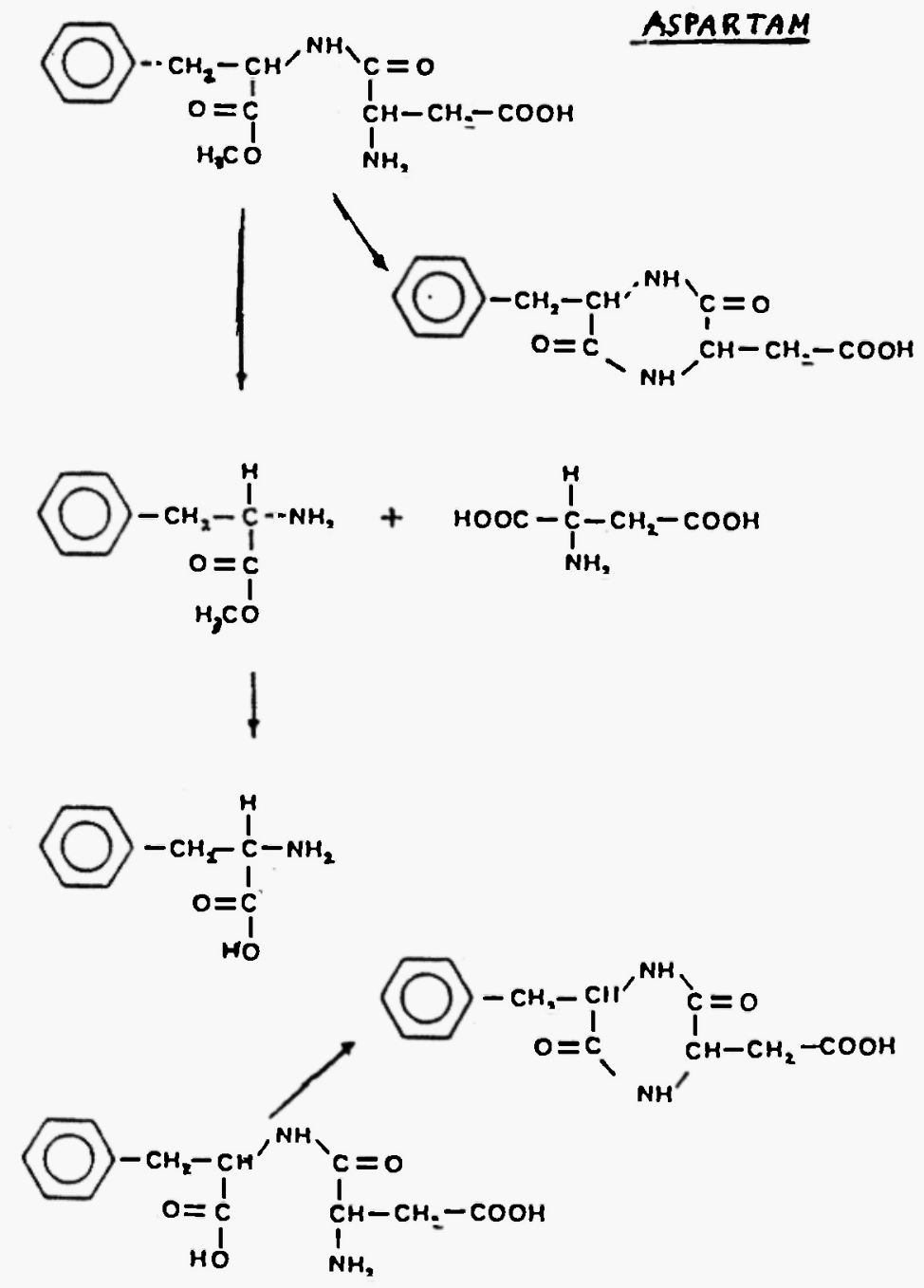

Fig. 2. 


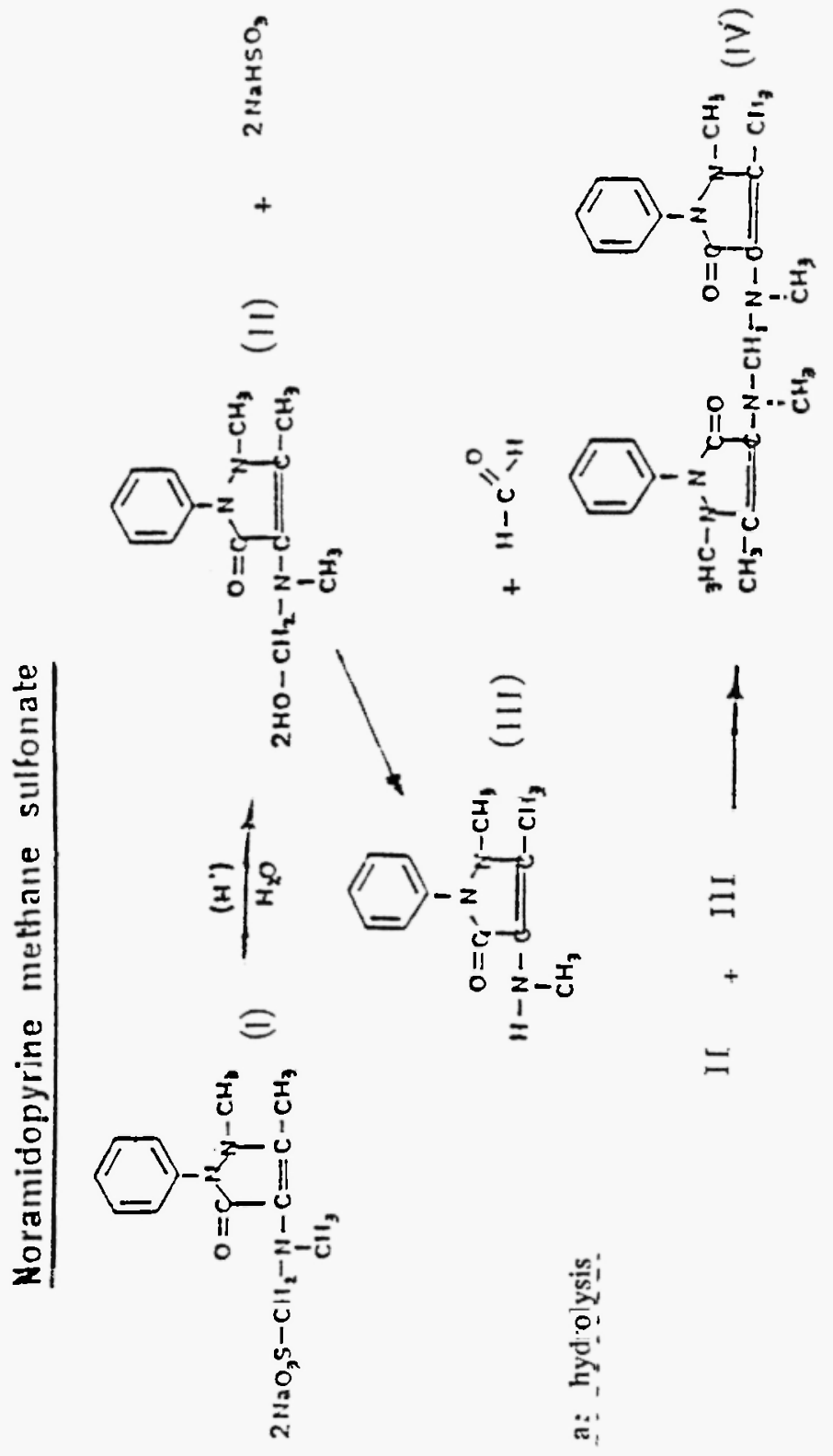



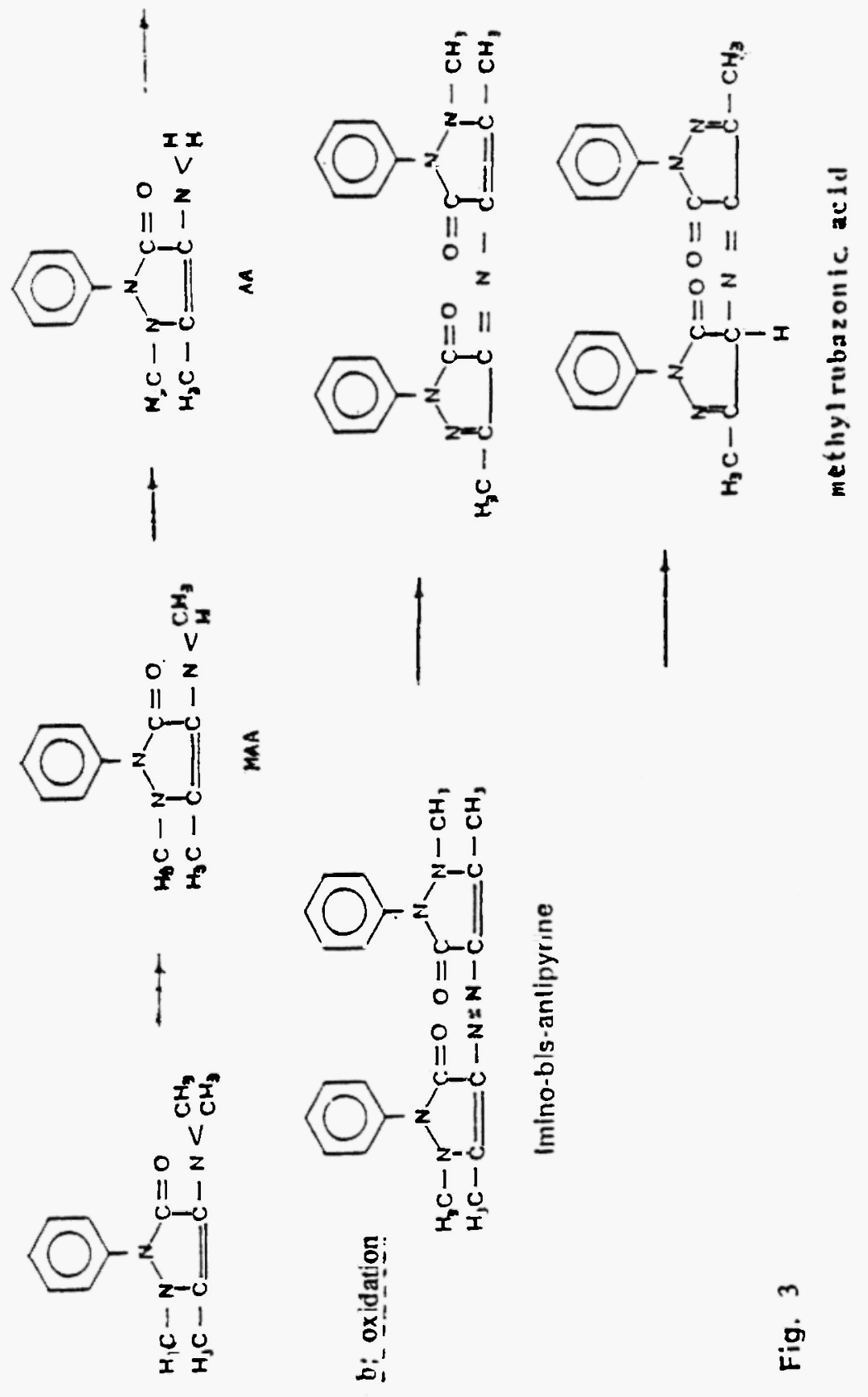
formulations in small proportions 91 to 2\%) maintains the equilibrium. Amino-4 antipyrine is only present in traces, not exceeding 0.05 per cent; its increase would correspond to a displacement of the equilibrium in the medium under the influence of an external factor (dilution, air, light).

2.3.4 Chelation reactions. Chemical chelation reactions are a cause of instability of organic molecules, going as far as to cause the disappearance of therapeutic properties. They are most often envisaged from the standpoint of protecting the stability, through the addition of a component or a chelator, in a medical form, such as tetracemate which masks the traces of metallic cations and suppresses their catalytic action. The formation of a chelate between a metal and the drug is often the cause of breakdown or instability of the molecule; this leads, as in the case of gentamycine or polymyxine $/ 16 /$, to the diminution, or even the suppression, of therapeutic activity. This fact should not be forgotten. Any time an organic molecule offers two functional groups capable of linking with a metal to form a 5- or 6-sided ring, chelation should be envisaged because of the new properties it might confer on the molecule; chelation takes place in the presence of traces of calcium, magnesium or zinc salts in the excipients. It can also be shown that such a reaction does not bring about the formation of a soluble chelate.

The formation of metal chelates often affects the excipients, a good many of which are applied elsewhere, for analytical purposes, as chelating agents. The possibility of these reactions could not be neglected when drug formulations are being prepared. For example, bismuth salts, with sorbitol or other polyols $/ 17 /$, form a chelate of known structure, water-soluble in an acidic medium, insoluble in water in a neutral medium /18/. The reaction is based on insoluble bismuth salts (e.g. nitrate, carbonate) in aqueous liquid media incorporating, in suspension or in solution, flavouring excipients or additives and ingredients of certain 
foodstuffs and drinks: lactic or citric acids; metaphosphates, polyols, etc. $/ 19,20 \%$.

3.

\section{REACTIVITY IN COMPLEX MIXTURES}

The study of the chemical reactions that develop between various constituents of a drug is often very difficult. To determine the stability of the drug it is essential to detect and identify the breakdown products. The analyst is confronted with many problems.

3.1 The convergence of reactions e.8. A+B $\rightarrow \mathrm{C}$ and $\mathrm{D}+\mathrm{E} \rightarrow \mathrm{F}$ may lead, by way of $C+F-\rightarrow X$, to an unexpected product. The formation of nitrosamines in drugs and food can result from the appearance in these media, through chemical reactions, of secondary amines and nitrous acid. Abdul Baki and Roth $21 /$ have shown the production of $\mathrm{N}$-nitrosodimethylamine from the secondary amine coming from promethazine and sodium nitrite.

Theoretically, nitrosamines require a secondary amine and a nitrous derivative for their formation; essentially, in a slightly acid aqueous medium or in the gas phase, the nitrous anhydride $\mathrm{N}_{2} \mathrm{O}_{3}$ acts as an $\mathrm{NO}^{+}$ion donor

$$
\underset{\mathrm{R}}{\mathrm{R}}=\stackrel{\delta^{-\delta^{+}}}{\mathrm{NH}}+\stackrel{\delta^{+}}{\mathrm{NO}}-\stackrel{\delta^{-}}{\mathrm{NO}_{2}} \rightarrow-\rightarrow \quad \stackrel{\mathrm{n}}{\mathrm{R}}=\mathrm{N}-\mathrm{NO}+\mathrm{HNO}_{2}
$$

Primary amines, if they are able to yield nitrosamines, lead to the formation of unstable products (responsible for mutagenic activity in vivo)

$$
\begin{aligned}
& \mathrm{R}-\mathrm{NH}_{2}+\mathrm{NO}^{+} \rightarrow \quad \mathrm{n}=\mathrm{N}-\mathrm{NO}+\mathrm{H}^{+} \\
& \rightarrow \mathrm{R}-\mathrm{N}^{+} \equiv \mathrm{N} \text { diazoalcene } \quad+\mathrm{H}_{2} \mathrm{O}
\end{aligned}
$$


Tertiary amines can give rise to quaternary derivatives with $\mathrm{NO}^{+}$

$$
\begin{aligned}
& \mathbf{R}_{1} \\
& \mathbf{R}_{2} \\
& \mathbf{R}_{3}
\end{aligned} . \mathrm{N}+\mathrm{NO}-\mathrm{NO}_{2}-\cdots \quad \begin{aligned}
& \mathbf{R}_{1} \\
& \mathbf{R}_{2} \\
& \mathbf{R}_{3}
\end{aligned} \quad \mathrm{~N}^{+}-\mathrm{NO}+\mathrm{NO}_{2}
$$

These derivatives are unstable and lead to the elimination of a radical, in the form of an aldehyde, and the formation of hyponitrous acid and a secondary amine.

It is doubtless by means of an analogous mechanism $/ 22 /$ that the thiourames (vulcanization acceleration agents for elastomers) can lead to the production of nitrosamines. Nitrous anhydride is formed by the partial oxidation of these derivatives to nitrosamines, at high temperatures in industrial operations. These compounds have been found in nipples and stoppers of injection solutions; they should not be confused with the supposedly nontoxic diaryl derivatives used as additives. The search for products not deliberately added but appearing in a pharmaceutical formulation as a result of a convergent reaction, is a very complex aspect of the study of stability. The presence of these unforseen components can be attributed to multiple reactions. The analyst must be vigilant to foresee these reactions and avoid the transformations. He must, therefore, take every advantage of his knowledge of organic chemical reactions and functional organic analysis.

The study of purity is, in itself, a factor of stability; traces of inorganic impurities (copper, iron, etc.) can engender catalytic breakdown of organic molecules.

In the same way, the stability of haloperidol is known and is described in Analytical Profiles of Drugs, by Klaus Florey 23/, this compound reacts with 5-(hydroxymethyl)-2 furfuraldehyde which is possibly present as an impurity in anhydrous lactose.

3.2 The study of the reactivity of organic components is not limited to solution phenomena; it deals also with reactions that 
occur in the absence of solutions or in dry or anhydric preparations. In fact, it cannot be asserted that the reaction occurs in the absence of solvent. the manufacture of pharmaceutical formulations may involve the use of solvents that are evaporated during drying; also certain excipients retain not insignificant amounts of water (up to $20 \%$ in the case of amidon). This "hidden" water in the constituents may become liberated during manufacture, causing, as a result of liquefaction or humidification, the reaction of the components present.

Thus, as was shown above, certain pharmaceutical excipients, such as polysorbates, hydrogenated oils, polyoxyethylene glycols and, in a general way, the excipients in all ointment formulations, have considerable dissolving power, the dissolution of the components in the medium causes the reaction to start. Their dissolving power is even the cause of incompatibility with plastic materials and significant exchanges between the recipient and its contents.

3.3 The effectiveness of the additives needed to maintain stability by blocking reactions or degradation is related to their often marked reactivity and to that of other components present. The study of chemical transformation is essential to avoid errors or the use of poorly active or even sometimes useless substances. Sodium bisulphite, for example, protects ascorbic acid from oxidation; in contrast, the breakdown of thiamine by sodium bisulphite restricts the use of this antioxidant /24/.<smiles>CC1=C(CCO)SCN1Cc1c(C)cnc(C)c1N</smiles>

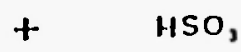<smiles>Cc1ncc(CS(=O)(=O)[O-])c(N)n1</smiles> 
It is not sufficient to show that oxidation has occurred and to identify the mechanism of the reaction. The choice of an antioxidant depends on the equilibrium potential between the redox mechanisms of the oxidizable compound and the antioxidant $125 \%$. The optimum concentration of the antioxidant - not to be confused with upper limit allowed by law - the synergism of mixtures of antioxidants, their consumption during storage, and the minimum concentration required to ensure its effectiveness up to the end of the product's shelf life, are determined by reactivity studies which justify and guarantee effectiveness.

3.4 Functional group analysis and prediction of stability. The methods of functional group analysis can be applied to the determination of drug stability. The reaction of ascorbic acid on amino-acids fixes their concentration under various conditions. The presence of these two types of compounds may be the cause of incompatibilities in the colouring of drugs. this fact was observed by C. Majcherczyck $126 /$ and made it possible to determine the reaction mechanism and to produce a diagram analogous to that for the reaction between amino-acids and ninhydrin. Ascorbic acid, Fig. 4 (I), is oxidized to dehydroascorbic acid in the lactone form (II) and finally a duplication of the molecule is obtained, Fig. 4 (III). The compound has been isolated in its structure determined by NMR and IR spectrometry.

The signal from the carbon atom carrying the side chain of the ascorbic acid is straightened out $(72.9 \mathrm{ppm}$ and $75.6 \mathrm{ppm})$ relative to that observed in the spectrum of ascorbic acid (74.9 ppm).

The displacements of carbon atoms 5 and 6 are identical, but the corresponding peaks are very broad; this indicates a steric constraint reducing the free rotation of the side chain $\mathrm{CHOH}-\mathrm{CH}_{2} \mathrm{OH}$ and confirms the envisaged structure.

The IR spectrophotometric analysis of the compound shows:

- a band at $1740 \mathrm{~cm}^{-1}$ characterizing the vibration of the $\mathrm{C}=0$ valency bond characteristic of unsaturated $\gamma$ lactones. 
- a wide band at $1500-1700 \mathrm{~cm}^{-1}$ corresponding to the absorption of the vibrations of the deformation of double bonds $\mathrm{C}=\mathrm{O} \quad \mathrm{C}=\mathrm{N} \quad \mathrm{C}=\mathrm{C}$.

- a band at 1180-1200 $\mathrm{cm}^{-1}$ characteristic of the deformations of the $\mathrm{C}-\mathrm{O}$ and $\mathrm{C}-\mathrm{N}$ groups.

- a band at 1500-1700 $\mathrm{cm}^{-1}$ confirms the presence of animine $(\mathrm{C}=\mathrm{N})$ group that is too much surrounded by other atoms to be detectable by the NMR of ${ }^{13} \mathrm{C}$.

The laboratory oxidation of organic compounds by hydrogen peroxide is a convenient means of studying stability and predicting breakdown $127,28 /$.

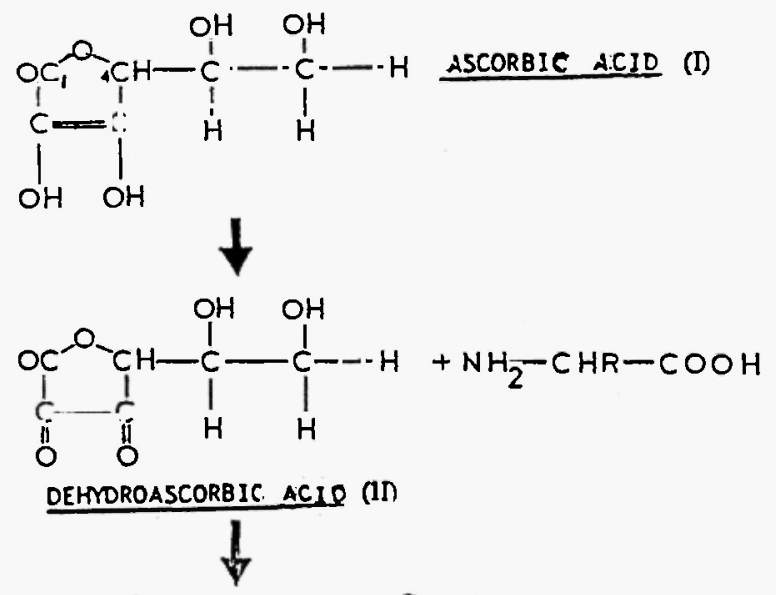<smiles>CN=Cc1cc(O)oc1C(O)CO</smiles><smiles>[O-]c1ccc(O)c(O[CH][C@@H](O)[C@H](O)CO)c1</smiles>

(III)

Fig. 4. 
The study of the mild oxidation of sulphamides has been carried out with a view to determining the exact structure of the oxidation products using spectroscopic analytical techniques, and the kinetics of oxidation. The objective of this is to transpose this study to pharmaceutical products to be put on sale, and to study their stability. Four sulphamides - sulphanilamide, sulphamethoxazole, sulphadoxine and probenecide - were oxidized by $20 \%$ hydrogen peroxide in $0.5 \mathrm{~N}$ sulphuric acid at room temperature under light. The products were separated in a silicone column then purified and crystallized.

The method of study used to define the structure of the products is based on:

- Elemental analysis

- UV spectroscopy

- IR spectroscopy

- NMR spectroscopy of ' $\mathrm{H}$ and ${ }^{13} \mathrm{C}$

- Mass spectrometry

The ultra-violet spectroscopy is carried out in acidic and alkaline media. Depending on the hypochrome or bathochorme displacements, the appearance or disappearance of $\mathrm{NH}_{2}$ or $\mathrm{OH}$ groups may be followed.

Infra-red spectroscopy in potassium bromide indicates the characteristics of the valency vibration bands and of the deformation of $\mathrm{NH}_{2}$ and $\mathrm{SO}_{2}$ groups. The appearance of supplementary bands allows the transformation of the original molecules to be determined.

$N M R$ spectroscopy of ' $\mathrm{H}$ or ${ }^{13} \mathrm{C}$ provides information on the environments of the ${ }^{\prime} \mathrm{H}$ and the ${ }^{13} \mathrm{C}$ as a function of the value of the increments revealed by the chemical displacement read from the spectrum.

Mass spectrometry is carried out by electron bombardment for sufficiently stable oxidation products (azoxy). In the case of more labile oxidation products (diol fraction), chemical ionization with $\mathrm{NH}_{3}$ gives, with good precision, the molecular weight of the compound. 
These procedures allow identification and determination of the structure of the main products, as follows:

1. Sulphanilamide

One diol derivative

One azoxy derivative

\section{SULFANILAFIDE}
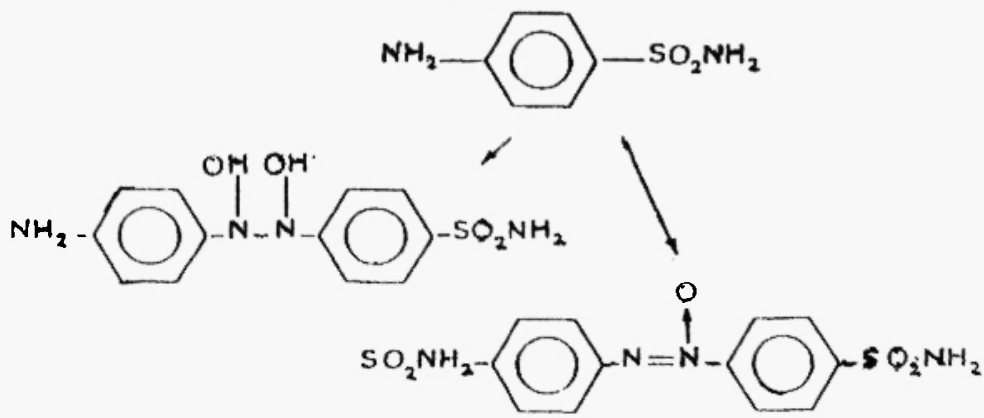

2. Sulphamethoxazole

Two major products:

a nitro derivative

an azoxy derivative
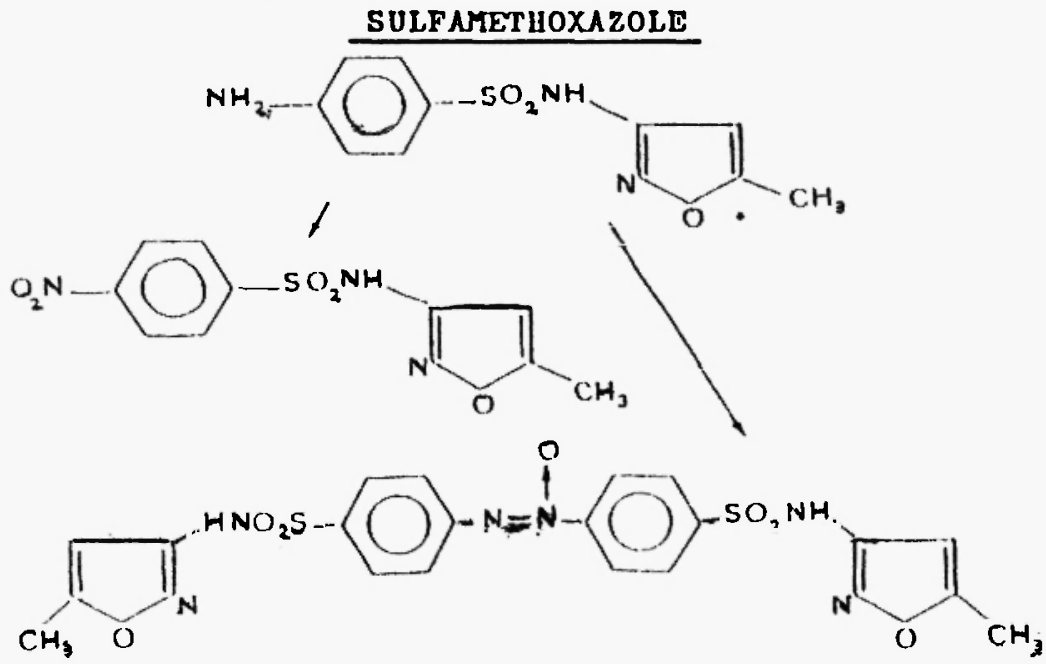
3. Sulphadoxine

Two products:

a nitro derivative

a hydroxylamino derivative

SULFADOXINE
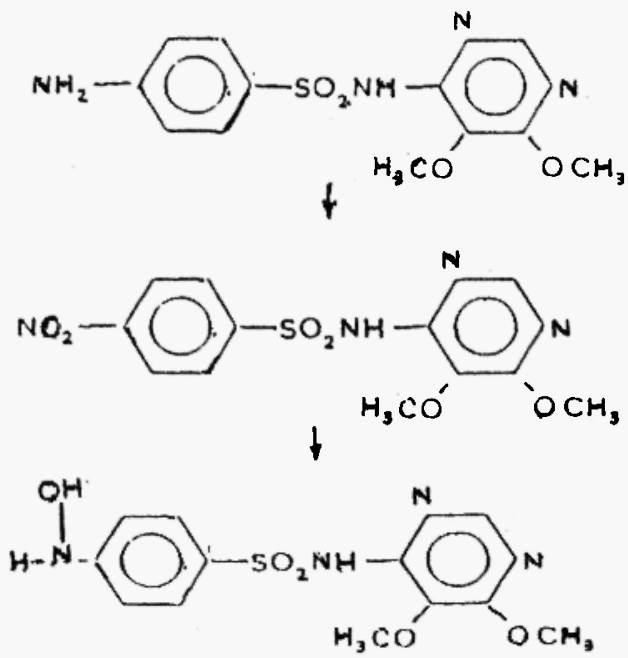

The kinetics werc determincd using HPLC with inverse phase polarity.

The kinetics are of zero order.

In conclusion, the stability of a drug rests on a in-depth study of the physical, physico-chemical, and chemical properties; in other words, on the rcactivity of the organic compounds. The identification of compounds formed in laboratory transformations under various conditions, and reactions between the components, constitutes the prior study to studies of the kinetics, forecasts of shelf life and the fixing of the date of validity. These studies precede the prevention of breakdown by means of appropriate additives and explain the transformation. The study of drug stability is, therefore, based on detailcd analytical 
research into the validity of the methods and a solid knowledge of their limitations. These results carry an unquestionable and adequate guarantee of chemical quality and safety of use.

\section{REFERENCES}

1. PELLERIN F., Pure and Appl. Chem. 1975, 44, 579.

2. PELLERIN F. et CHANON N., Sci. Techn. Pharm. 1981, 10, 341.

3. PESEZ M., Publication de l'O.M.S. (W.H.O.) - Pharm. 79495 (W.H.O. Geneve 1980).

4. GARRETT E.R., Pharmacie industrielle, No. 79, 1966.

5. CHANON N., These Doct. Sciences, Rouen 1981.

6. LAFOREST J., THUILLIER G., J. Heter. Chem. 1977, 14, 793-796.

7. FOX B.L., SALOMAN S., GOLDSCHMIDT M., Technical report AFML TR 72, 4, University of Dayton, Janvier 1972.

8. BARTON H., Die Pharmazie, 1980, 35 (3) 155.

9. HEYES W.F., SALMON J.R., J. Chromalo. 1980, No. 194.

10. MAJCHERCZYK C., These 3eme Cycle - F. PELLERIN's Laboratory University Paris XI.

11. BAYLOCQ D., MAJCHERCZYK C. et PELLERIN F., Talanta 1986, 33, 1, 85-89.

12. PELLERIN F., LETAVERNIER J.F., Ann. Pharm. Fr. 1973, 31, 3, 161.

13. PELLERIN F., CHANON N., DUMITRESCU D., Colloque INSERM Paris 1983. (INSERM Editor).

14. ONO S., ONISHI R., KAWAMURA K., J. Pharm. Soc. Japan 1966, 86, 11 .

15. PECHTOLD F., Arzneim. Forsch. 1964, 14, 258-474-1056.

16. NEWTON B.A., J. Gen. Microbio. 1954, 10, 491.

17. VANINO L., HARTL F., J. Praks. Chem. 1960, 74, 145.

18. PELLERIN F., MANCHERON D., Ann. Pharm. Fr. 1967, 25, 797.

19. PELlERIN F., GOULlE J.P., DUMITRESCU D., Ann. Pharm. Fr. $1977,35,281$. 
20. PELLERIN F., GOULLE JP., DUMITRESCU D., Bull. Acad. Nat. de Medecine 1976, 160, 268.

21. ABDUL-BAKI AHMAD, Arch. Pharm. (Weinheim Germ.) 1978, 311 (9) 775-779.

22. EL HASSAF, M. HAMON et F. PELLERIN, Ann. Falsif. et Exp. Chim. (1982) 75 No. 804, 29-34.

23. FLOREY K., Analytical profiles of drugs (volume 1-a 19) Academic Press.

24. "Sulphites as Food Additives" Foad Trade. Rev. 1976, 45 (2) 76.

25. PELLERIN F., BAYLOCQ D., Labo-Pharma 1980, 340, 535.

26. BAYLOCQ D., MAJCHERCZYK C. et PELLERIN F., Talanta 1983, 30, 72.

27. De SOUZA A., BAYLOCQ D. and PELLERIN F., Congres du GAMS, Paris 1987.

28. De SOUZA A., BAYLOCQ D. and PELLERIN F., Talanta 1988, 35, 875. 